\title{
Diodelike Asymmetric Transmission of Linearly Polarized Waves Using Magnetoelectric Coupling and Electromagnetic Wave Tunneling
}

\author{
Mehmet Mutlu, ${ }^{1, *}$ Ahmet E. Akosman, ${ }^{1}$ Andriy E. Serebryannikov, ${ }^{2}$ and Ekmel Ozbay ${ }^{1}$ \\ ${ }^{1}$ Department of Electrical and Electronics Engineering, Nanotechnology Research Center, Bilkent University, 06800 Ankara, Turkey \\ ${ }^{2}$ Department of Electrical Engineering, E-3, Hamburg University of Technology, D-21071 Hamburg, Germany
}

(Received 7 August 2011; revised manuscript received 18 January 2012; published 24 May 2012)

\begin{abstract}
An asymmetric, reciprocal, diffraction-free transmission of linearly polarized waves in a new diodelike, three-layer, ultrathin, chiral structure is studied theoretically and experimentally. The exploited physical mechanism is based on the maximization of the cross-polarized transmission in one direction due to the polarization selectivity dictated by the peculiar eigenstate combination, which is efficiently controlled by the electromagnetic tunneling through the metallic subwavelength mesh sandwiched between these layers. Simulation and microwave experiment results demonstrate a nearly total intensity transmission at normal incidence in one direction and a small intensity transmission in the opposite direction.
\end{abstract}

DOI: 10.1103/PhysRevLett.108.213905

PACS numbers: 41.20.Jb, 42.25.Ja, 78.20.-e

Simultaneous breaking of time reversal and spatial inversion symmetries has been considered as necessary for nonreciprocal transmission in volumetric structures, enabling the obtaining of $\omega(\mathbf{k}) \neq \omega(-\mathbf{k})$ while involving anisotropic constituents [1,2]. The possibility of nonreciprocal light propagation owing to the symmetry of the parity-time operator has also been studied, for example, in two-channel structures with a properly chosen symmetry of the complex refractive index [3]. At the same time, there were attempts to achieve asymmetric but still reciprocal transmission while using conventional isotropic, linear and low-loss or lossless materials. Among them, the asymmetric transmission of linearly polarized waves in diffractive nonsymmetrical volumetric gratings based on photonic [4] and sonic [5] crystals, or made of ultralow-index materials [6], and in nonsymmetrical metallic gratings supporting surface plasmons $[7,8]$ should be mentioned. The asymmetric transmission of circularly polarized waves has been demonstrated at normal incidence in thin structures composed of meta-atoms [9-12]. A thin structure composed of three-dimensional meta-atoms without any rotational symmetry has been suggested, which allows asymmetric transmission for an arbitrary, also linear, polarization of the incident electromagnetic wave [13].

In this Letter, we propose a new, ultrathin, three-layer structure made of isotropic and linear materials, which enables a diodelike asymmetric transmission of certain linearly polarized, normally incident waves. Nearly total transmission is demonstrated in a cross-polarization regime in one direction, while the transmission in the opposite direction at the same polarization of the incident wave is blocked. Possible applications include microwave and optical isolation, integrated photonic circuits, ultrafast information processing and optical interconnects [14]. To the authors' knowledge, diodelike asymmetric transmission of linearly polarized waves at normal incidence has not been observed before. In fact, it requires the diagonal components and one of the off-diagonal components of the Jones matrix to be zero, while the only nonzero element must be approximately unity for maximum efficiency. These requirements are not easily achievable also in a chiral structure, without the support of additional physical mechanisms, in our case the utilization of elliptical eigenstates and the tunneling of electromagnetic waves.

The exploited physical mechanism is based on the combination of the coupling of electric and magnetic fields in mutually rotated split ring resonator (SRR) pairs [15] and electromagnetic wave tunneling through a metallic subwavelength mesh [16]. Tunneling is an exciting phenomenon that utilizes the potential of nonpropagating waves in transmission. Unlike the previous experience of utilizing the tunneling effect [16], we perform an $A B C$ stacking-type instead of an $A B A$-type. Layers $A$ and $C$ are, in fact, the same layers that are rotated by $\pm \pi / 2$ with respect to each other. Layer $B$ is a subwavelength mesh that acts as a negative permittivity medium throughout the interested frequency range independent of the polarization. Because of the inclusion of layer $B$, the phase difference between the transmission coefficients of the eigenwaves of the $A B C$ stack may be properly optimized, yielding a strong modification in the transmission, i.e., enhancement of one of the two off-diagonal (cross-polarized) components of the Jones matrix $\left(T_{x y}\right)$ and suppression of all the others $\left(T_{x x}, T_{y x}, T_{y y}\right)$. As a result, strongly asymmetric, diodelike transmission takes place for the incident waves that are linearly polarized in the $x$ or $y$ directions. Similar to Refs. [9-13], asymmetric transmission in the suggested structure is a completely reciprocal phenomenon.

One of the main ideas behind the proposed design is choosing two layers, which do not exhibit $C_{4}$ symmetry, as layers $A$ and $C$. In addition, these layers must be chosen such that they exhibit strong optical activity when brought together. Accordingly, we choose the separate layers of the metamaterial structure given in Ref. [17] as layers $A$ and $C$. 
Adopting the Jones matrix formalism, we relate the electric field amplitudes of incident $\left(I_{x}, I_{y}\right)$ and transmitted $\left(T_{x}, T_{y}\right)$ fields [13], for the $+z$ (forward) propagation as follows:

$$
\left(\begin{array}{c}
T_{x}^{f} \\
T_{y}^{f}
\end{array}\right)=\left(\begin{array}{ll}
T_{x x}^{f} & T_{x y}^{f} \\
T_{y x}^{f} & T_{y y}^{f}
\end{array}\right)\left(\begin{array}{c}
I_{x}^{f} \\
I_{y}^{f}
\end{array}\right)=\left(\begin{array}{cc}
\delta & \gamma \\
\beta & \delta
\end{array}\right)\left(\begin{array}{c}
I_{x}^{f} \\
I_{y}^{f}
\end{array}\right)
$$

Then, for the $-z$ (backward) propagation, we have

$$
\left(\begin{array}{c}
T_{x}^{b} \\
T_{y}^{b}
\end{array}\right)=\left(\begin{array}{cc}
\delta & -\beta \\
-\gamma & \delta
\end{array}\right)\left(\begin{array}{c}
I_{x}^{b} \\
I_{y}^{b}
\end{array}\right)
$$

According to (1) and (2), if $I_{y}^{f}=I_{y}^{b}=0$ (x-polarized incident wave) and $\delta=0$, all transmitted energy corresponds to the nondiagonal (cross-polarized) elements, leading to a strongly asymmetric transmission if these elements are significantly different in magnitude. Diodelike asymmetric transmission also occurs when $I_{x}^{f}=$ $I_{x}^{b}=0$. However, in this case, the direction of the strong transmission should be reversed. Next, we will demonstrate that almost all of the incident wave energy can be converted to that of the cross-polarized transmitted wave for one of the incidence directions, while it is largely reflected for the opposite direction, so that a diodelike behavior occurs. In the ideal case, one must obtain $\gamma=1$ and $\beta=0$. For the purposes of this study, we seek for the regimes with $\gamma \approx 1$ and $\beta \ll \gamma$.

The unit cell of the proposed structure that is assumed to be periodic in the transverse plane is depicted in Fig. 1. It is composed of three distinct layers that are referred to as layers $A, B$, and $C$. Figures 1 (a) and 1(b) shows layer $A$ and layer $C$, respectively. They both contain four U-shaped SRRs and show the lack of $C_{4}$ symmetry due to the involvement of the SRRs of different dimensions. The arrangement of layers $A$ and $C$, and the scheme of stacking are presented in Fig. 1(c) together with the geometry of
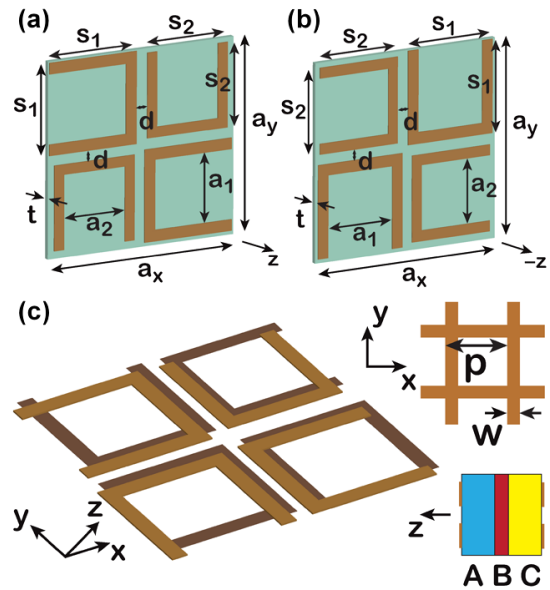

FIG. 1 (color online). Geometries of (a) layer $A$ and (b) layer $C$. (c) Arrangement of $A$ and $C$ with respect to each other along with the mesh geometry and the stacking scheme. layer $B$. In the arrangement of $A$ and $C$, the SRRs of the same dimensions are paired together. Layer $B$ is positioned symmetrically between $A$ and $C$. The geometric parameters for layer $B$ are given by $w=0.5 \mathrm{~mm}$ and $p=$ $2.56 \mathrm{~mm}$. The geometric parameters of both layers $A$ and $C$ are given by $s_{1}=6 \mathrm{~mm}, a_{1}=4.6 \mathrm{~mm}, s_{2}=5.52 \mathrm{~mm}$, $a_{2}=4.23 \mathrm{~mm}, d=0.64 \mathrm{~mm}, t=1 \mathrm{~mm}$ and $a_{x}=a_{y}=$ $12.8 \mathrm{~mm}$. Teflon dielectric substrates with a relative dielectric permittivity of 2.1 and a loss tangent of 0.0002 are utilized for the layers $A$ and $C$. Layer $B$ is considered to be a stand-alone metallic structure, with a thickness of $20 \mu \mathrm{m}$. The metal utilized for the structure of the SRRs and the mesh is copper with a $20 \mu \mathrm{m}$ thickness. The total thickness of the structure is $2.06 \mathrm{~mm}$, which corresponds to $\lambda / 21$ at $7 \mathrm{GHz}$ while $a_{x} / \lambda \approx 0.3$.

We started the analysis with numerical simulations using CST Microwave Studio (Computer Simulation Technology, AG, Germany), by using periodic boundary conditions along the $x$ and $y$ directions and an open boundary condition for the $z$ direction, while the excitation source is an $x$-polarized plane wave. Then, in order to experimentally verify the diodelike transmission, we fabricated the structure with the dimension of 23 by 23 unit cells. The experiment was conducted using two standard horn antennas facing each other at a $50 \mathrm{~cm}$ distance. The sample is positioned in the middle between the antennas. The transmission coefficient measurements are performed using a HP-8510C network analyzer (Agilent Technologies, USA). For the experimental characterization of the structure, the transmitted fields for the forward and backward propagating incident waves are studied in terms of the transmission coefficients $T_{x x}$ and $T_{y x}$.

As the first step, we studied the responses of the single layers, $A, B$, and $C$. Figures 2(a) and 2(b) show $\left|T_{x x}\right|$ and $\left|T_{y x}\right|$, respectively. We see in Fig. 2(a) that layer $A$ is resonant at $7.7 \mathrm{GHz}$ whereas the resonance frequency is $7 \mathrm{GHz}$ for layer $C$. Field distributions (not shown) reveal that at the resonance frequency, the responses of the layers
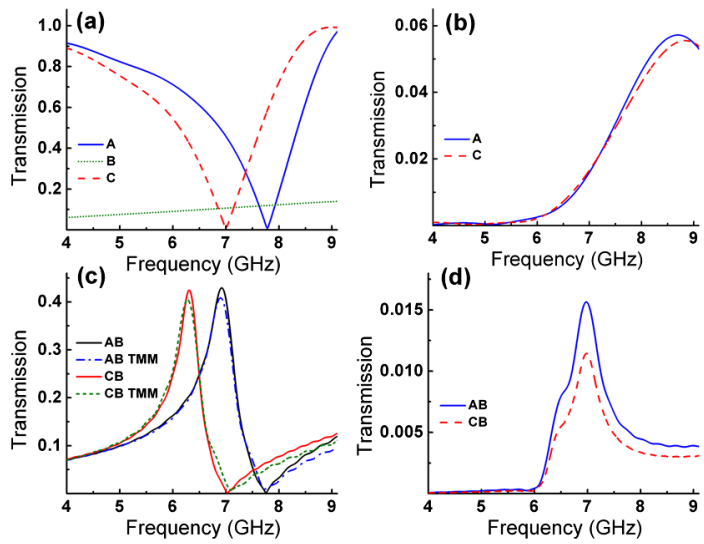

FIG. 2 (color online). $\left|T_{x x}\right|$ for (a) separate and (c) double layers, and $\left|T_{y x}\right|$ for the same (b) separate and (d) double layers. 
are governed by the SRRs that have slits parallel to the direction of the electric field. In the case of layer $C$, such SRRs are larger, which implies that their resonance frequency is lower. Figure 2(b) shows that the $\left|T_{y x}\right|$ values of layers $A$ and $C$ show maxima at around $8.5 \mathrm{GHz}$. However, $\left|T_{y x}\right|$ does not exceed 0.06 , indicating that the coupling between electric and magnetic fields in the transverse plane is relatively weak. The small difference in $\left|T_{y x}\right|$ values for layers $A$ and $C$ is caused by the weak symmetry breaking of the substrate [18].

The numerical results of the $A B$ and $C B$ cases are presented in Fig. 2(c) for $\left|T_{x x}\right|$ and in Fig. 2(d) for $\left|T_{y x}\right|$. Using Fig. 2(c), we observe transmission bands at 6.2 and $7 \mathrm{GHz}$ for the $A B$ and $C B$ cases, respectively. Moreover, the frequencies of zero transmissions are not modified by adding layer $B$. Noting that $\left|T_{y x}\right|$ is negligible compared to $\left|T_{x x}\right|$ in these cases, we can approximately model the transmission of $A B$ and $C B$ utilizing the transfer matrix method (TMM), while assuming the eigenwaves to be linearly polarized, i.e., similarly to Ref. [16]. Using the effective medium theory (EMT), for $x$-polarized incident waves, we characterize the separate layers as homogenous dielectric slabs with $\varepsilon_{A}=5.67+252 /\left(7.78^{2}-f^{2}\right), \varepsilon_{B}=$ $3.65-55.7^{2} / f^{2}$, and $\varepsilon_{C}=6.33+286 /\left(7^{2}-f^{2}\right)$ where $f$ is the frequency in GHz. The effective thickness required to reproduce the simulated magnitude and phase values is $1.22 \mathrm{~mm}$ for layers $A$ and $C$, and $1.5 \mathrm{~mm}$ for layer $B$. The results obtained from the TMM calculations are shown in Fig. 2(c) and are in excellent agreement with the simulations. At the same time, we observe a peak at $7 \mathrm{GHz}$ for $\left|T_{y x}\right|$; see Fig. 2(d). In order to theoretically describe $\left|T_{y x}\right|$ using EMT, one should determine the elliptically polarized eigenwaves for layers $A$ and $C$ and retrieve the effective permittivity for each eigenwave. Afterwards, TMM calculations should again be performed.

Thereafter, we simulated the composite structure with the $A B C$ stacking-type. The numerical results reveal that increasing the distance between the layers decreases $\left|T_{x y}\right|$ as a result of the decreased evanescent-wave coupling. Thus, one should not leave any air gaps. The numerical and experimental results that demonstrate the diodelike asymmetric transmission phenomenon are shown in Fig. 3. According to these results, the frequency of the diodelike transmission is $7 \mathrm{GHz}$. In Fig. 3(c), it is observed that $\left|T_{y x}\right|$ approaches unity for the backward propagating waves, whereas $T_{x x}$ is close to zero for both propagation directions. In turn, Fig. 3(a) shows that only $10 \%$ of the incident field is transmitted when the structure is excited by a forward propagating wave. To be exact, $\left|T_{x x}\right|$ is approximately 0.03 , and $\left|T_{y x}\right|$ is 0.1 and 0.98 for the forward and backward propagating waves, respectively. These parameters provide $1.09 \%$ intensity transmission for the forward propagation and $96.1 \%$ intensity transmission for the backward propagation. These values correspond to the contrast of $88(19.5 \mathrm{~dB})$, which is obtained

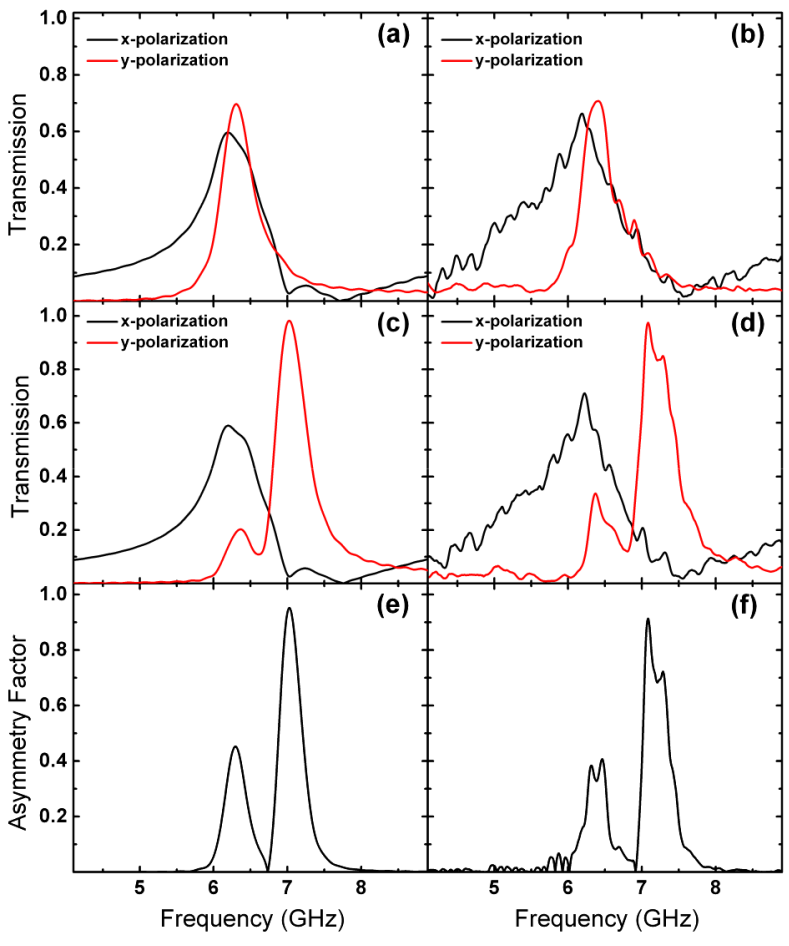

FIG. 3 (color online). Numerical and experimental transmission spectra for the $A B C$ stack, for $x$-polarized (a), (b) forward and (c), (d) backward propagating waves; (e), (f) numerical and experimental asymmetry factor.

as the ratio of the two transmitted intensities. For the backward propagation, the magnetic field distribution inside the composite structure (not shown) that is similar to the one given in Ref. [16] reveals the occurrence of the electromagnetic tunneling due to the high magnetic fields (see Fig. 2 in Ref. [16]). The transmission of the composite $A B C$ structure can also be modeled using EMT as we have done for the $A B$ and $C B$ cases. However, in the composite case, the eigenwaves are elliptically polarized, so that significant effort is needed to accurately apply the EMT framework. Instead, subsequently, we will describe the behavior of the structure in terms of the eigenstate interpretation.

According to Fig. 3, the experiment results are in good agreement with the numerical results, except for a slight shift of the frequency of the maximal asymmetry (7.1 GHz). The possible reasons for this shift can include (i) the difference of the effective permittivity of the Teflon substrate from the simulations, (ii) the small differences in substrate and metal thicknesses, and (iii) the finite size effects. Experimental data show that $\left|T_{x x}\right|=0.141$ and $\left|T_{y x}\right|=0.171$, for a forward propagating wave. These transmission coefficients yield $4.9 \%$ intensity transmission at 7.1 GHz. On the other hand, for the backward propagation, we obtain that $\left|T_{x x}\right|=0.112$, whereas $\left|T_{y x}\right|=0.974$, which corresponds to $96 \%$ intensity transmission. Hence, the experimental contrast is $19.6(13 \mathrm{~dB})$. 
In this context, the asymmetry factor is defined as $\left|T_{x x}^{b}\right|^{2}+\left|T_{y x}^{b}\right|^{2}-\left|T_{x x}^{f}\right|^{2}-\left|T_{y x}^{f}\right|^{2}$. We demonstrate the asymmetry factor of the structure for an $x$-polarized incident wave numerically and experimentally in Figs. 3(e) and 3(f), respectively. In particular, one can see two peaks of the asymmetry factor that are positioned at 6.3 and $7 \mathrm{GHz}$. Such behavior is quite expectable while these two frequencies correspond to the transmission peaks of $\left|T_{y x}\right|$, where the transmitted intensity difference is maximized as a result of the increasing difference between the forward and backward $\left|T_{y x}\right|$ values. The simulation results state a 0.45 and 0.95 asymmetry factor at 6.3 and $7 \mathrm{GHz}$, respectively. Experimental results show great agreement in terms of the asymmetry factor, which is now of 0.38 at $6.3 \mathrm{GHz}$ and 0.91 at $7.1 \mathrm{GHz}$. The direction of diodelike transmission is reversed for a $y$-polarized wave as is obvious from Eqs. (1) and (2). The asymmetry factor is strongly dependent on the incident polarization and has its maximum for the $x$ - and $y$-polarized waves. On the other hand, the asymmetry factor is zero for an incident wave that is linearly polarized at $45^{\circ}$, meaning complete symmetry in transmission.

For the achievement of diodelike asymmetric transmission, the Jones matrix descriptions in Eqs. (1) and (2) state that $T_{x x}=T_{x y}=T_{y y}=0$ and $T_{y x}=-1$ must be achieved for backward propagation. However, using the present $A B C$-type stacking approach, the transmission matrix for backward propagation is achieved as

$$
T=\left(\begin{array}{cc}
0 & -\alpha e^{j \varphi} \\
-1 & 0
\end{array}\right)
$$

where $\alpha=0.1$. As it will be demonstrated subsequently, $\varphi$ does not affect the magnitudes of the transmission coefficients and therefore, its value is not explicitly given. Afterwards, the eigenwaves of the $A B C$ structure are found as [19]

$$
\mathbf{i}_{1}=\left(\begin{array}{c}
1 \\
\alpha^{-1 / 2} e^{-j \varphi / 2}
\end{array}\right), \quad \mathbf{i}_{2}=\left(\begin{array}{c}
1 \\
-\alpha^{-1 / 2} e^{-j \varphi / 2}
\end{array}\right) .
$$

The eigenstates are orthogonal and counter-rotating elliptically polarized waves. We can define the transmitted waves as $T_{1} \mathbf{i}_{1}$ and $T_{2} \mathbf{i}_{2}$ when $\mathbf{i}_{1}$ and $\mathbf{i}_{2}$ are incident on the structure, respectively. Here, $T_{1}$ and $T_{2}$ are complex transmission coefficients for the eigenwaves. Next, by decomposing an $x$-polarized incident field in terms of the eigenwaves of the system, we obtain

$$
T\left(\begin{array}{l}
1 \\
0
\end{array}\right)=\frac{1}{2}\left(T_{1} \mathbf{i}_{1}+T_{2} \mathbf{i}_{2}\right)=\left(\begin{array}{c}
(1 / 2)\left(T_{1}+T_{2}\right) \\
\left(\alpha^{-1 / 2} / 2\right) e^{-j \varphi / 2}\left(T_{1}-T_{2}\right)
\end{array}\right) .
$$

Thus, for the purpose of achieving $\left|T_{x x}\right|=0$, we need $T_{2}=-T_{1}$. In other words, the two counter-rotating eigenwaves must be transmitted with a phase difference of $\pi$. At this point, the phase difference is a critical parameter. The subwavelength mesh in the structure acts as a necessary degree of freedom that enables the obtaining of the desired phase difference between the transmitted eigenwaves. Then, $\left|T_{y x}\right|$ is found as $\alpha^{-1 / 2}\left|T_{1}\right|$ and equal to unity at $7 \mathrm{GHz}$ and, therefore, for this frequency, $\left|T_{1}\right|=\left|T_{2}\right|=$ 0.316. Electromagnetic tunneling becomes evident as a result of the constructive interference of the transmitted eigenwaves in the $y$ direction. Next, we decompose a $y$-polarized incident wave in terms of the eigenwaves as follows:

$$
T\left(\begin{array}{l}
0 \\
1
\end{array}\right)=\frac{e^{j \varphi / 2}}{2 \alpha^{-1 / 2}}\left(T_{1} \mathbf{i}_{1}-T_{2} \mathbf{i}_{2}\right)=\left(\begin{array}{c}
\left(\sqrt{\alpha} e^{j \varphi / 2} / 2\right)\left(T_{1}-T_{2}\right) \\
(1 / 2)\left(T_{1}+T_{2}\right)
\end{array}\right) .
$$

In that case, $\left|T_{y y}\right|$ is equal to zero at $7 \mathrm{GHz}$. On the other hand, $\left|T_{x y}\right|$ is given by $\alpha^{1 / 2}\left|T_{1}\right|$ and equal to 0.1 . Moreover, the ratio between $\left|T_{y x}\right|$ and $\left|T_{x y}\right|$ is found as $\alpha^{-1}$, as was expected from Eq. (3). Note that, due to Lorentz reciprocity $T_{x y}^{b}=-T_{y x}^{f}$. Therefore, we conclude that the presented diodelike asymmetric transmission originates from the common effect of the two counter-rotating elliptically polarized eigenwaves that are transmitted through the structure with a phase difference of $\pi$. The phase difference is of crucial importance since it determines the magnitudes of the co- and cross-polarized transmission. One should choose the eigenwaves such that the magnitude of their component along the $y$ direction $\left(\alpha^{-1 / 2}\right)$ should be as large as possible for maximizing the transmission asymmetry. The calculations based on the Jones matrix formalism reveal that, if $\alpha \ll 1$ is achieved, any structure exhibiting the transmission of the elliptically polarized eigenstates with a phase difference of $\pi$ would exhibit diodelike asymmetric transmission. It is noteworthy that the modification of the mesh thickness can decrease the asymmetry factor significantly as a consequence of modifying the phase difference between the transmitted eigenstates.

In a recent study, we have shown the construction of a $90^{\circ}$ polarization rotator by combining chirality and electromagnetic tunneling, which benefits from the tunneling of the circularly polarized eigenstates with a phase difference of $\pi$ [20]. In contrast to the present study, the structure is $C_{4}$ symmetric, which leads to circularly polarized eigenstates and, therefore, a polarization independent and symmetric response. The proposed design in this Letter shows the peculiarity of introducing unity transmission with $90^{\circ}$ polarization rotation for a certain polarization, whereas the cross polarization is largely reflected, which is allowed due to the breaking of the $C_{4}$ symmetry to obtain orthogonal elliptically polarized eigenstates that can be optimized to interfere either constructively or destructively at different polarization directions. The enabling of the polarization selective interference and unequal decomposition of the $x$ - and $y$-polarized waves in terms of the eigenwaves [as shown in Eqs. (5) and (6)] are the premier 
effects that allow for the observation of the diodelike asymmetric transmission phenomenon.

To summarize, we demonstrated a diodelike asymmetric transmission for normally incident $x$ - and $y$-polarized waves in an electrically thin reciprocal structure. In addition to the planar ultrathin design, the usage of isotropic and linear materials only makes this and similar structures the promising candidates for practical applications. The backward-to-forward transmission contrast of the structure at the operating frequency $7 \mathrm{GHz}$ is 19.5 and $13 \mathrm{~dB}$ according to the simulation and experiment results, respectively. The asymmetry factor at this frequency is 0.95 for the simulation results and 0.91 for the experiments. The ideas of the suggested approach are promising for future designs at the terahertz and optical frequency ranges.

This work is supported by the projects DPT-HAMIT, ESFEPIGRAT, EU-N4E, NATO-SET-181, and TUBITAK under Projects No. 107A004, No. 107A012, and No. 109E301. A.E. S. thanks DFG for partial support under Projects No. SE1409-2/1 and No. SE1409-2/2. One of the authors (E. O.) also acknowledges partial support from the Turkish Academy of Sciences.

*mutlu@ee.bilkent.edu.tr

[1] A. Figotin and I. Vitebsky, Phys. Rev. E 63, 066609 (2001).

[2] Z. Yu, Z. Wang, and S. Fan, Appl. Phys. Lett. 90, 121133 (2007).

[3] C.E. Rüter, K. G. Makris, R. El-Ganainy, D. N. Christodoulides, M. Segev, and D. Kip, Nature Phys. 6, 192 (2010).

[4] A. E. Serebryannikov, Phys. Rev. B 80, 155117 (2009).
[5] X.-F. Li, X. Ni, L. Feng, M.-H. Lu, C. He, and Y.-F. Chen, Phys. Rev. Lett. 106, 084301 (2011).

[6] A.E. Serebryannikov and E. Ozbay, Opt. Express 17, 13335 (2009).

[7] W. Sun, Q. He, J. Hao, and L. Zhou, Opt. Lett. 36, 927 (2011).

[8] S. Cakmakyapan, H. Caglayan, A. E. Serebryannikov, and E. Ozbay, Appl. Phys. Lett. 98, 051103 (2011).

[9] R. Singh, E. Plum, C. Menzel, C. Rockstuhl, A. K. Azad, R. A. Cheville, F. Lederer, W. Zhang, and N. I. Zheludev, Phys. Rev. B 80, 153104 (2009).

[10] E. Plum, V. A. Fedotov, and N. I. Zheludev, Appl. Phys. Lett. 94, 131901 (2009).

[11] V.A. Fedotov, P. L. Mladyonov, S. L. Prosvirnin, A. V. Rogacheva, Y. Chen, and N. I. Zheludev, Phys. Rev. Lett. 97, 167401 (2006).

[12] V. A. Fedotov, A. S. Schwanecke, N. I. Zheludev, V. V. Khardikov, and S. L. Prosvirnin, Nano Lett. 7, 1996 (2007).

[13] C. Menzel, C. Helgert, C. Rockstuhl, E.-B. Kley, A. Tünnermann, T. Pertsch, and F. Lederer, Phys. Rev. Lett. 104, 253902 (2010).

[14] X. Hu, C. Xin, Z. Li, and Q. Gong, New J. Phys. 12, 023029 (2010).

[15] N. Liu, H. Liu, S. Zhu, and H. Giessen, Nature Photon. 3, 157 (2009).

[16] L. Zhou, W. Wen, C. T. Chan, and P. Sheng, Phys. Rev. Lett. 94, 243905 (2005).

[17] M. Mutlu, A. E. Akosman, A. E. Serebryannikov, and E. Ozbay, Opt. Express 19, 14290 (2011).

[18] S. I. Maslovski, D. K. Morits, and S. A. Tretyakov, J. Opt. A 11, 074004 (2009).

[19] C. Menzel, C. Rockstuhl, and F. Lederer, Phys. Rev. A 82, 053811 (2010).

[20] M. Mutlu and E. Ozbay, Appl. Phys. Lett. 100, 051909 (2012). 